OPEN ACCESS

Edited by:

Chunjie Jiang

University of Pennsylvania,

United States

Reviewed by:

Xuexin Yu,

University of Texas Southwestern

Medical Center, United States

Shanlan Mo,

Shenzhen Institutes of Advanced

Technology, Chinese Academy

of Sciences (CAS), China

Wei Chen,

Beihang University, China

*Correspondence:

Xiaoman B

bixiaoman@hainmc.edu.cn

Zhonglin Mu

muzhonglin2@sina.com

Kongning $L$

likongning@hainmc.edu.cn

tThese authors have contributed equally to this work and share first authorship

Specialty section:

This article was submitted to

Computational Genomics,

a section of the journal

Frontiers in Genetics

Received: 30 June 2021

Accepted: 17 August 2021

Published: 17 September 2021

Citation

Xu D, Ding S, Cao M, Yu X, Wang H, Qiu D, Xu Z, Bi X, Mu Z and Li K (2021) A Pan-Cancer Analysis of

Cystatin E/M Reveals Its Dual

Functional Effects and Positive

Regulation of Epithelial Cell in Human

Tumors. Front. Genet. 12:733211.

doi: 10.3389/fgene.2021.733211

\section{A Pan-Cancer Analysis of Cystatin E/M Reveals Its Dual Functional Effects and Positive Regulation of Epithelial Cell in Human Tumors}

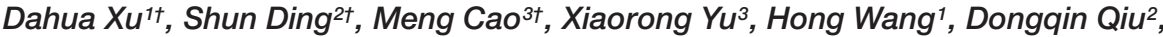 \\ Zhengyang $\mathrm{Xu}^{2}$, Xiaoman $\mathrm{Bi}^{{ }^{*}}$, Zhonglin $\mathrm{Mu}^{2 *}$ and Kongning $\mathrm{Li}^{1{ }^{1 *}}$
}

${ }^{1}$ Key Laboratory of Tropical Translational Medicine of Ministry of Education, College of Biomedical Information and Engineering and Cancer Institute of the First Affiliated Hospital, Hainan Medical University, Haikou, China, ${ }^{2}$ Department of Otolaryngology, Head and Neck Surgery, The First Affiliated Hospital, Hainan Medical University, Haikou, China, ${ }^{3}$ College of Bioinformatics Science and Technology, Harbin Medical University, Harbin, China

Cystatin E/M (CST6), a representative cysteine protease inhibitor, plays both tumorpromoting and tumor-suppressing functions and is pursued as an epigenetically therapeutic target in special cancer types. However, a comprehensive and systematic analysis for CST6 in pan-cancer level is still lacking. In the present study, we explored the expression pattern of CST6 in multiple cancer types across $\sim 10,000$ samples from TCGA (The Cancer Genome Atlas) and 8,000 samples from MMDs (Merged Microarray-acquired Datasets). We found that the dynamic expression alteration of CST6 was consistent with dual function in different types of cancer. In addition, we observed that the expression of CST6 was globally regulated by the DNA methylation in its promoter region. CST6 expression was positively correlated with the epithelial cell infiltration involved in epithelial-to-mesenchymal transition (EMT) and proliferation. The relationship between CST6 and tumor microenvironment was also explored. In particular, we found that CST6 serves a protective function in the process of melanoma metastasis. Finally, the clinical association analysis further revealed the dual function of CST6 in cancer, and a combination of the epithelial cell infiltration and CST6 expression could predict the prognosis for SKCM patients. In summary, this first CST6 pan-cancer study improves the understanding of the dual functional effects on CST6 in different types of human cancer.

Keywords: CST6, pan-cancer, DNA methylation, epithelial cell, EMT, tumor microenvironment, prognosis

\section{INTRODUCTION}

Cystatin E/M (also known as CST6) is a member of the cystatin superfamily that performs physiological inhibitors of lysosomal cysteine proteases through forming high-affinity reversible complexes (Turk and Bode, 1991). The dysfunction of CST6 contributed to the alterations in proteolysis of tissue architecture, which might accelerate the spread of cancer cells (Shridhar et al., 2004; Keppler, 2006). Increasing evidence has demonstrated the dual functional effects of CST6 in cancer progress (Lalmanach et al., 2021). For instance, the overexpression of CST6 
could rescue mice from bone metastasis by suppressing proliferation, migration, and invasion (Jin et al., 2012). Moreover, the loss of CST6 expression has been observed in lung and cervical cancer, and its recovery expression resulted in growth suppression in culture (Zhong et al., 2007; Veena et al., 2008). In contrast to the protective function, Hosokawa et al. (2008) found that the upregulated expression of CST6 promoted tumor growth in vitro and in vivo in pancreatic ductal adenocarcinoma. CST6 was also shown overexpressed in triple-negative breast cancer and oral cancer, facilitating the tumor metastatic process (Vigneswaran et al., 2003; Li et al., 2018). Collectively, CST6 played crucial and disparate roles in the pathogenesis and development of cancer. However, a comprehensive research about the expression pattern and functional effects of CST6 in pan-cancer level is still lacking.

As an important epigenetically regulatory factor, DNA methylation has been implicated in the dysfunction of CST6. One study has shown that the hypermethylation status of CST6 promoter resulted in CST6 deficiency in glioma tumor-initiating cells, while the promoter was hypomethylated in normal brain tissues (Qiu et al., 2008). The aberrant methylation and downregulated expression of CST6 were also found in breast cancer patients, and the expression could reactivate after DNA demethylating agent treatment (Ai et al., 2006; Schagdarsurengin et al., 2007). However, the relationship between the expression and the DNA methylation of CST6 in pan-cancer level remains unclear.

Numerous studies have shown the important roles of tumor microenvironment in cancer therapy and diagnosis (Wu and Dai, 2017; Hinshaw and Shevde, 2019). CST6 is an epithelium-specific protease inhibitor with essential roles in epidermal differentiation (Zeeuwen et al., 2010). Zhang et al. (2004) found that CST6 was consistently expressed in normal human breast epithelial cells, while it was decreased in breast invasive carcinoma samples, and the expression of CST6 was associated with cell proliferation, migration, and invasion. The CST6 promoter was found highly methylated in cfDNA of breast cancer plasma cells but not in healthy samples (Chimonidou et al., 2013). Moreover, IL-17A, an immunotherapy targeting, could affect keratinocyte differentiation by regulating the expression of CST6 (Sato et al., 2020). However, there has been limited research that comprehensively explored the relationship between CST6 and tumor microenvironment in pancancer level.

In this study, we performed a systematic evaluation of the expression pattern of CST6 across cancer types from TCGA and MMDs. Consistent with the known dual role of CST6, we found that there was a broad spectrum of CST6 expression across cancer types. Through DNA methylation analysis, we found that the expression of CST6 was globally regulated by the methylation level of its promoter region. Moreover, the expression of CST6 was related to epithelial cell infiltration, EMT, and proliferation. Finally, the association between the CST6 expression and patient survival was also investigated. Our first pan-cancer study for
CST6 provided novel insights into its dual function in the development of cancer.

\section{MATERIALS AND METHODS}

\section{Analysis of Gene Expression}

We entered CST6 in the "Gene_DE" module of the TIMER2 website $^{1}$ (Li et al., 2021) and explored the expression of CST6 between different tumors and adjacent normal tissues in TCGA items. For some tumors with no normal sample or the number of normal tissue specimens was less than 5, we used the "Expression Analysis-Expression DIY" module of the GEPIA2 2 to compare the expression level of CST6 between tumor tissues and GTEx (Genotype-Tissue Expression) datasets (Tang et al., 2019). The gene with $|\log 2 \mathrm{FC}|>1$ and $p$-value $<0.05$ was considered as significantly differentially expressed by the ANOVA method. In addition, we used the pathological stage module and subtype filter module in GEPIA2 to obtain the expression of CST6 in different tumors at different stages and different subtypes. In order to verify the expression pattern of CST6 in different cancer types, we collected gene expression data of more than 8,000 samples from 11 cancers (Supplementary Table 1; Lim et al., 2019). To avoid differences between platforms to the greatest extent, only the dataset generated from the Affymetrix Human Genome U133 Plus 2.0 array was processed to develop the MMDs dataset. All datasets were processed uniformly through RMA normalization, and batch effect were corrected through the Combat $\mathrm{R}$ package (Leek et al., 2012). Moreover, the protein level of CST6 between tumor and normal tissue was obtained from the CPTAC analysis module of the UALCAN portal ${ }^{3}$ (Chandrashekar et al., 2017). An external validation dataset was obtained with GEO accession GSE46517, which included 73 metastatic and 31 primary melanoma patients.

\section{DNA Methylation Analysis}

We downloaded the gene expression and HM450 DNA methylation profiles across cancer types of TCGA PanCancer (PANCAN) cohort through UCSC Xena. ${ }^{4}$ The full name, abbreviation, and sample number of cancer types for TCGA are shown in Supplementary Tables 2, 3. The methylation level of CST6 was quantified by averaging the beta values of $\mathrm{CpGs}$ located in the promoter region (upstream $2 \mathrm{~kb}$ to TSS). Then, Wilcoxon rank test was used to identify differentially methylated CST6 between tumor and normal tissue. In addition, the correlation between DNA methylation and expression of CST6 was calculated using the Pearson correlation method. The results of correlation coefficient less than -0.3 and $p$-value $<0.05$ were identified as significant.

\footnotetext{
${ }^{1}$ http://timer.cistrome.org/

${ }^{2}$ http://gepia2.cancer-pku.cn/

${ }^{3}$ http://ualcan.path.uab.edu/analysis-prot.html

${ }^{4}$ http://xena.ucsc.edu/
} 


\section{Cystatin E/M-Related Gene Functional Enrichment Analysis}

The CST6-related genes were obtained from the "Similar Gene Detection" module of GEPIA2 by Pearson correlation method. We selected the datasets of all TCGA tumors and finally screened out the top 500 CST6-correlated genes. For a specific cancer type in TCGA and MMDs datasets, we used the Pearson correlation method to obtain CST6-related genes (Pearson correlation $>0.3$ ). To explore the potential biological function that CST6 regulated, we passed the CST6-related genes to Metascape 5 with the setting of species ("Homo sapiens") (Zhou et al., 2019).

\section{Estimation of the Relationship Between Cystatin E/M and Tumor Microenvironment, Epithelial-to-Mesenchymal Transition, and Tumor Proliferation}

In order to assess the infiltration levels of epithelial cells in diverse cancers, we downloaded the precalculated TCGA data from xCell, ${ }^{6}$ a method that yielded cell type enrichment score for 64 immune and stroma cell types, which included epithelial cell infiltration score (Aran et al., 2017).

We estimated the EMT score for an independent cancer type according to the method of Zhang et al. (2020). The epithelial and mesenchymal genes were obtained from a previous study (Mak et al., 2016). Then, the EMT score, which could reflect the EMT level for each sample, was calculated according to the following formula:

$$
\mathrm{S}_{E M T}=\sum_{i}^{N} \frac{M^{i}}{N}-\sum_{j}^{n} \frac{E^{j}}{n}
$$

where $\mathrm{N}$ represents the number of mesenchymal genes, and $\mathrm{n}$ represents the number of epithelial genes.

The expression level of the proliferation marker ki67 (MKI67) was used to reflect tumor proliferation in TCGA samples. Then, the correlation between CST6, cell infiltration, and proliferation score were estimated through Spearman's rank-order correlation method. The relationship between EMT score and CST6 was calculated by partial correlation through "ggm" $\mathrm{R}$ packages considering tumor purity as concomitant variable.

\section{Survival Prognosis Analysis}

The survival analysis of OS (overall survival) and RFS (disease-free survival) for CST6 in TCGA tumors were performed through GEPIA2. The median expression of CST6 was used to divide the patients into high-expression and low-expression groups. Then, the OS and RFS of these groups were compared by log-rank test. In addition, the survival interaction between CST6 expression and epithelial cell

${ }^{5}$ https://metascape.org/gp/index.html\#/main/stepl

${ }^{6} \mathrm{https}: / / \mathrm{xcell}$.ucsf.edu/ (positive genes obtained from xCell) was explored using siGCD ${ }^{7}$ (Cui et al., 2021).

\section{RESULTS}

\section{Gene Expression Analysis of Cystatin $\mathrm{E} / \mathrm{M}$ in the the Cancer Genome Atlas Datasets}

CST6 could serve as a biomarker for tumor diagnosis and play a dual functional effect across cancer types (Lalmanach et al., 2021). Previous studies of CST6 expression in cancer were limited to sample sizes and focused on a single cancer type. To comprehensively characterize the expression pattern of CST6, we first applied the "Gene_DE" module of TIMER2 portal for TCGA datasets. As shown in Figure 1A, the expression level of CST6 in bladder urothelial carcinoma (BLCA), breast invasive carcinoma (BRCA), cervical and endocervical cancer (CESC), cholangiocarcinoma (CHOL), colon adenocarcinoma (COAD), esophageal carcinoma (ESCA), rectum adenocarcinoma (READ), thyroid carcinoma (THCA), and uterine corpus endometrioid carcinoma (UCEC) is significantly higher than in normal samples, while the expression level of CST6 in kidney chromophobe (KICH), kidney clear cell carcinoma (KIRC), lung adenocarcinoma (LUAD), and lung squamous cell adenocarcinoma (LUSC) is significantly decreased than adjacent normal samples. Moreover, the patients with HPV infection showed a lower expression level of CST6 in head and neck squamous cell carcinoma (HNSC), and the patients with metastasis status showed a lower expression in skin cutaneous melanoma (SKCM).

Due to the limited normal sample size for several cancer types, we included the normal tissue of the GTEx datasets and evaluated the expression difference of CST6. As shown in Figure 1B, we found that the expression of CST6 was significantly different in CESC, ovarian serous cystadenocarcinoma (OV), pancreatic adenocarcinoma (PAAD), and SKCM. These results were consistent with the tumor-promoting function of CST6 in breast cancer ( $\mathrm{Li}$ et al., 2018), pancreatic cancer (Hosokawa et al., 2008), and papillary thyroid carcinoma (Oler et al., 2008). The tumor-suppressive function of CST6 has also been reported in lung cancer (Zhong et al., 2007), melanoma (Briggs et al., 2010), and renal cell carcinoma (Morris et al., 2010), which agreed with the loss expression of CST6 in these cancer types.

The results of the CPTAC dataset showed that the expression of CST6 total protein in BRCA and KIRC was lower than that of normal tissues (Figure 1C, $t$-test, $p$-value $<0.001$ ). To extend the expression pattern of CST6 with tumor pathological information, we applied the "Stage Plot" and "Subtype Filter" functions of GEPIA2. The expression of CST6 was related to the stage of BLCA, COAD, kidney papillary cell carcinoma (KIRP), OV, READ, SKCM, THCA, and uterine carcinosarcoma (UCS) (Figure 1D). In addition, the

\footnotetext{
${ }^{7}$ http://sigcd.idrug.net.cn/Home
} 


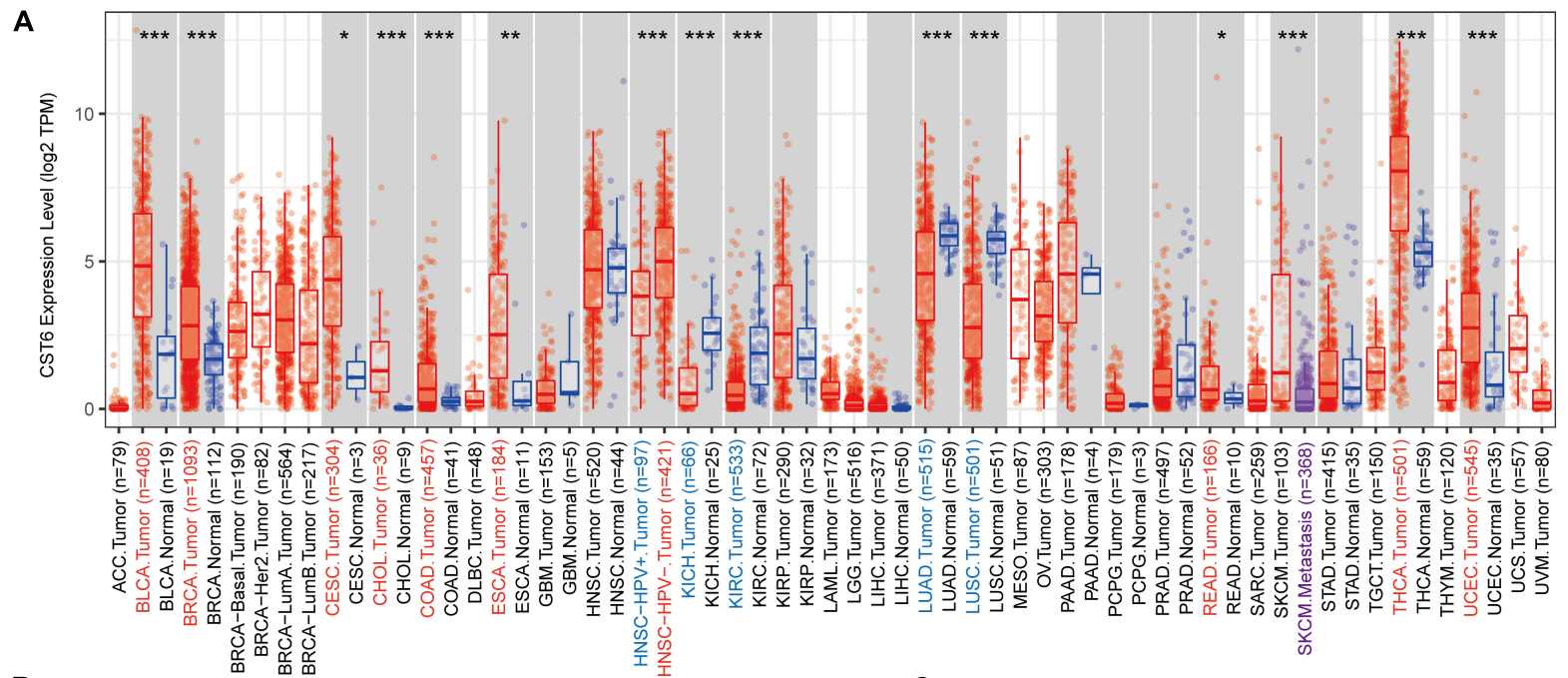

B

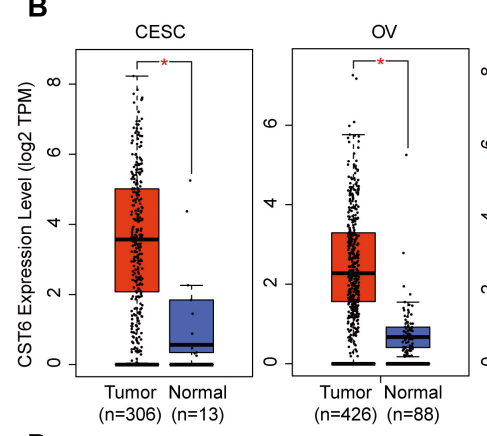

D
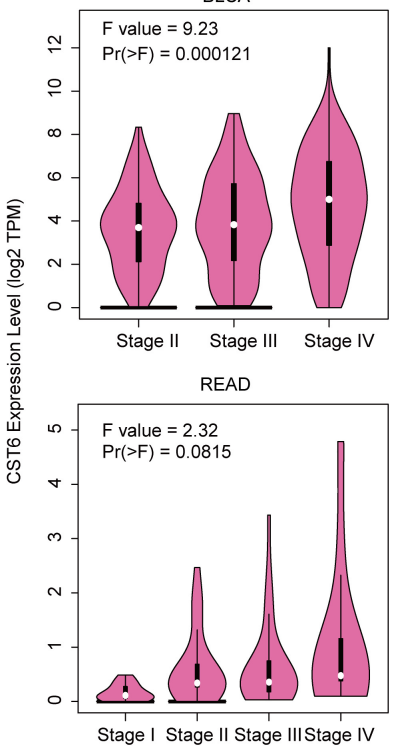

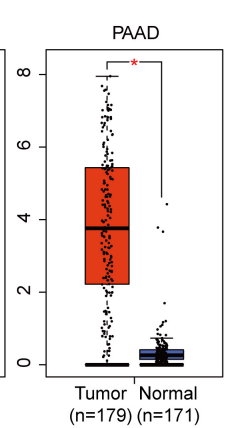

COAD

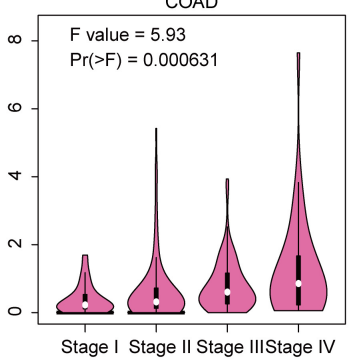

SKCM

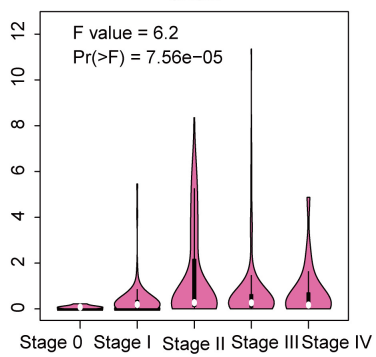

C

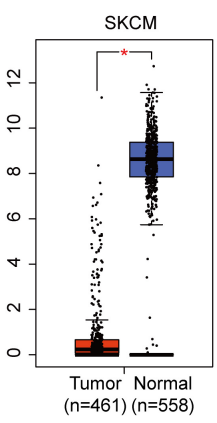

CPTAC_BRCA ***
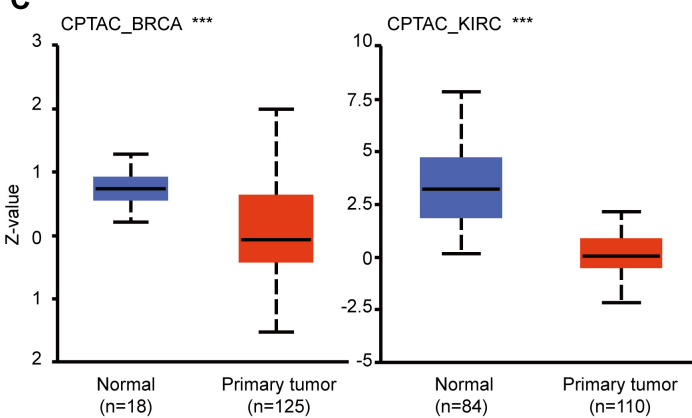

KIRP
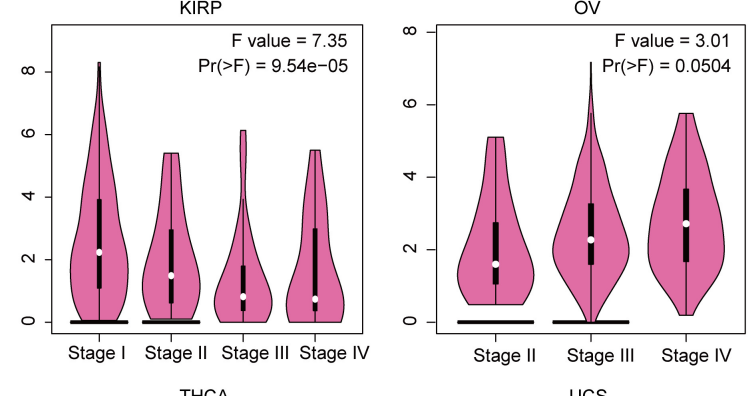

THCA
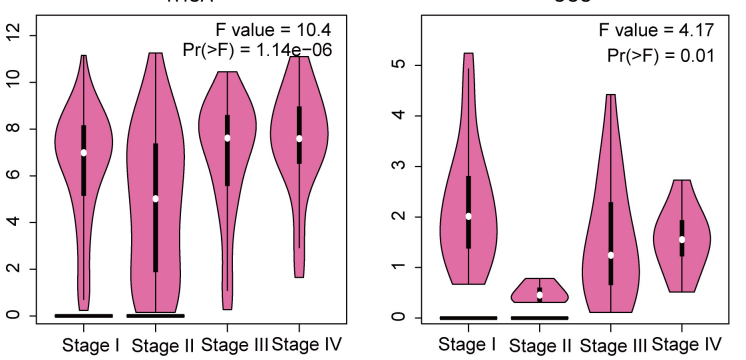

FIGURE 1 | Expression level of cystatin E/M (CST6) in different cancer types and pathological stages. (A) The expression level of CST6 in different cancer types from The Cancer Genome Atlas datasets. ${ }^{*} p<0.05 ;{ }^{* *} p<0.01 ;{ }^{* *} p<0.001$. The symbol with red represents upregulated, and the symbol with blue represents downregulated. (B) The expression level of CST6 in cervical and endocervical cancer (CESC), ovarian serous cystadenocarcinoma (OV), pancreatic adenocarcinoma (PAAD), and skin cutaneous melanoma (SKCM). The corresponding normal samples of the GTEx datasets were included. (C) The expression level of CST6 total protein for breast invasive carcinoma (BRCA) and kidney clear cell carcinoma (KIRC) cancer types in the CPTAC dataset. (D) The expression level of CST6 was associated with the pathological stages of bladder urothelial carcinoma (BLCA), colon adenocarcinoma (COAD), kidney papillary cell carcinoma (KIRP), OV, rectum adenocarcinoma (READ), SKCM, thyroid carcinoma (THCA), and uterine carcinosarcoma (UCS) cancer types. 


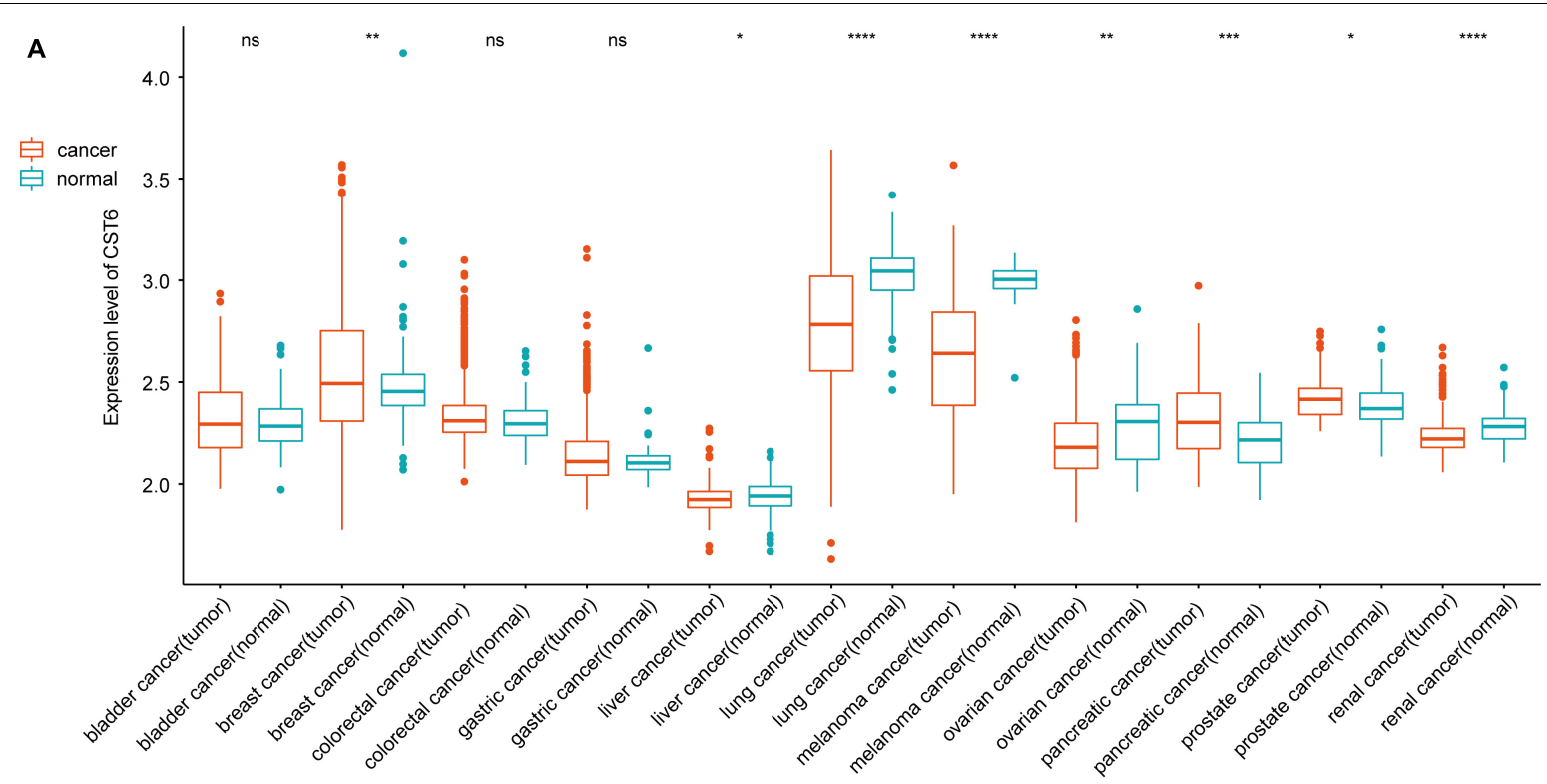

B

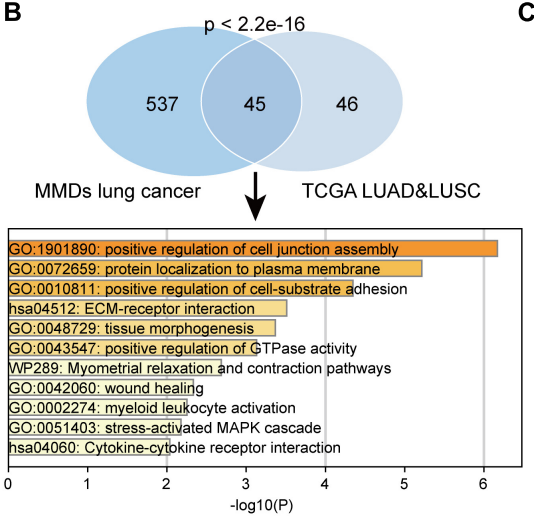

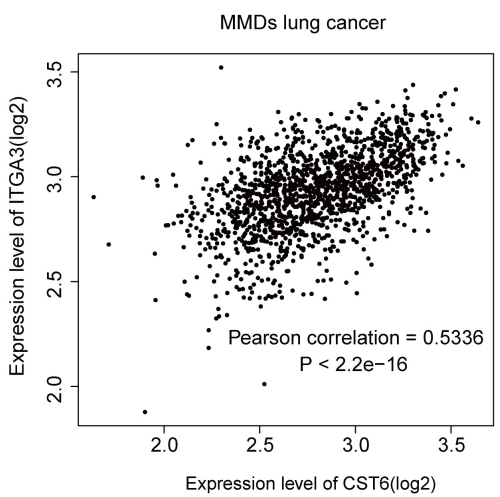

D

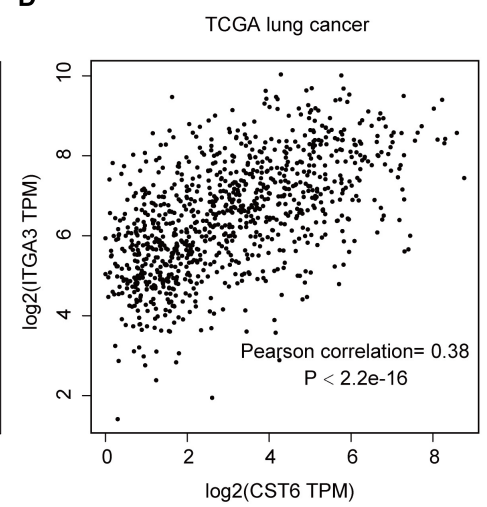

FIGURE 2 | Expression level of CST6 in Merged Microarray-acquired Datasets (MMDs) datasets. (A) The expression level of CST6 in different cancer types from MMDs. ${ }^{*} p<0.05 ;{ }^{* \star} p<0.01 ;{ }^{* \star *} p<0.001 ;{ }^{* \star \star} p<0.0001$; ns, not significant. (B) The comparison and functional analysis of CST6-related genes between MMDs lung cancer and TCGA lung adenocarcinoma (LUAD) and lung squamous cell adenocarcinoma (LUSC) datasets. (C,D) The correlation between the expression of CST6 and integrin $\alpha 3$ (ITGA3) for lung cancer in MMDs and TCGA datasets.

expression of CST6 was significantly different in the subtypes of CESC, HNSC, KIRP, LUAD, LUSC, PAAD, and THCA (Supplementary Figure 1).

\section{Gene Expression Analysis of Cystatin $\mathrm{E} / \mathrm{M}$ in the Merged Microarray-Acquired Datasets}

To verify the expression pattern of CST6 across cancer types, we collected the MMDs of more than 7,000 samples from 11 cancers with a standard process. Particularly, we revealed that CST6 showed a higher expression in breast cancer, pancreatic cancer, and prostate cancer, while the expression level of CST6 significantly decreased in liver cancer, lung cancer, melanoma, ovarian cancer, and renal cancer (Figure 2A). We found that the expression pattern of CST6 was consistent in breast, pancreatic, lung, melanoma, and renal cancer between TCGA and MMDs datasets. The expression of CST6 was upregulated in BLCA, COAD, and READ for TCGA datasets, while no significantly differently expressed was observed in bladder and colorectal cancer for the MMDs. Moreover, the expression of CST6 was specifically dysregulated in liver, ovarian, and prostate cancer for the MMDs. Regarding the most downregulated cancer type in the two datasets, the coexpression genes of CST6 (Pearson correlation $>0.3$ ) were commonly shared between MMDs lung cancer and TCGA LUAD/LUSC datasets, showing a statistically significant overlap (Figure 2B, hypergeometric test, $p$-value $<2.2 \mathrm{e}-16)$. We also found that the overlap of CST6related genes was enriched in the tumor microenvironmentrelated processes (such as positive regulation of cell junction assembly and protein localization to the plasma membrane, Figure 2B).

In addition, integrin $\alpha 3$ (ITGA3) was the most related gene in the two datasets, which showed a positively correlation 

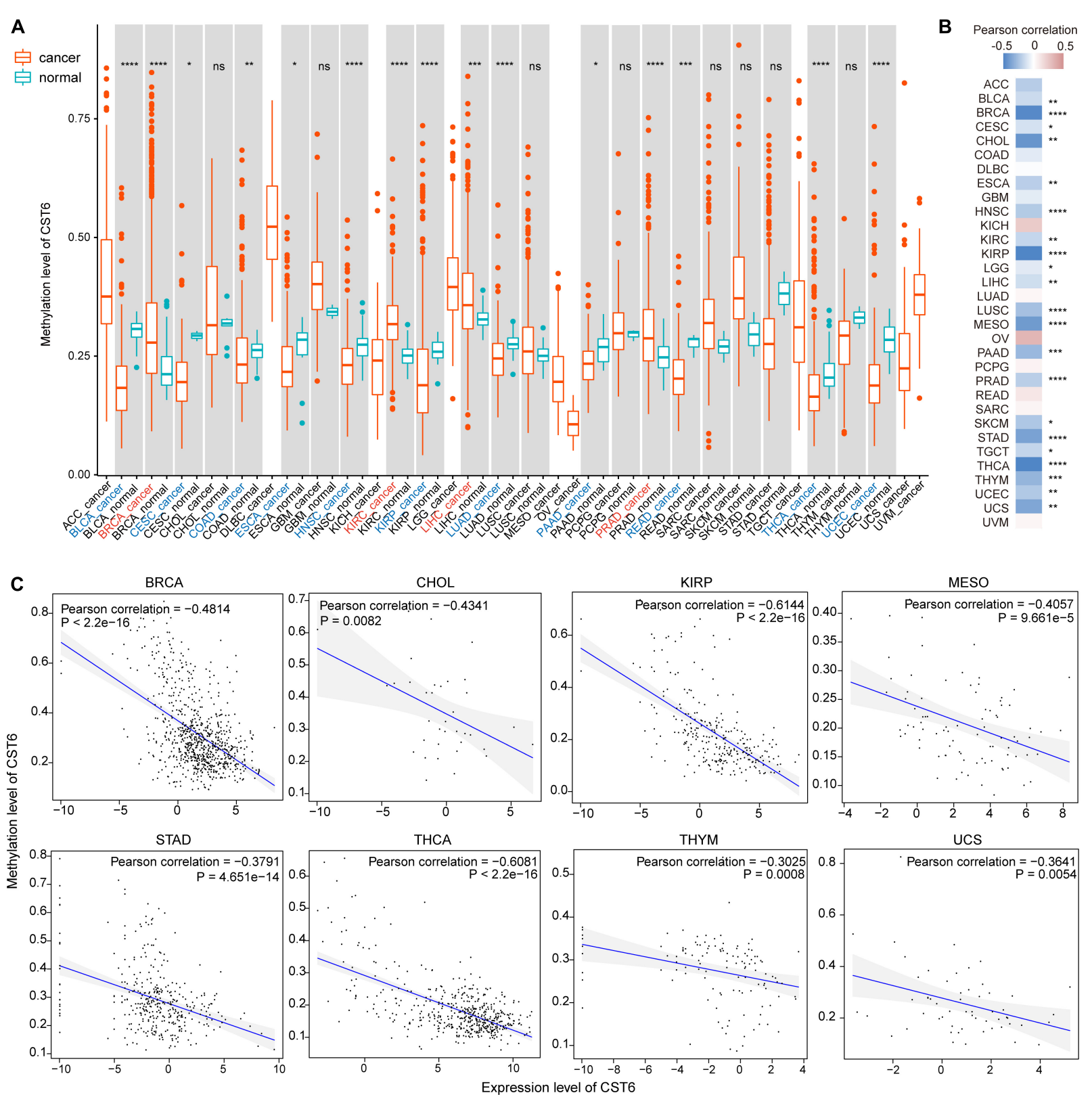

FIGURE 3 | The expression of CST6 was globally regulated by DNA methylation. (A) The DNA methylation level of CST6 in different cancer types from TCGA datasets. ${ }^{\star} p<0.05$; ${ }^{* \star} p<0.01 ;{ }^{* \star *} p<0.001 ;{ }^{* \star \star} p<0.0001$; ns, not significant. (B) The Pearson correlation coefficients between the expression and methylation level of CST6 in TCGA cancer types. (C) The correlation between the expression and methylation level of CST6 in BRCA, CHOL, KIRP, MESO, STAD, THCA, THYM, and UCS cancer types (Pearson correlation coefficient $<-0.3, p$-value $<0.01$ ).

with the expression of CST6 (Figures 2C,D). Moreover, a recent study revealed the important prognostic role of ITGA3 in patients with non-small cell lung cancer (Li et al., 2020). Thus, CST6 and ITGA3 may be potential therapeutic targets for lung cancer. Similar results were obtained in melanoma and renal cancer samples. We found that kallikreinrelated peptidase 7 (KLK7) and keratin 7 (KRT7) were the most related genes in melanoma and renal cancer separately
(Supplementary Figure 2). Aberrant expression of KLK7 has found to be related to melanoma aggressiveness by stimulating cell migration and adhesion (Delaunay et al., 2017; Haddada et al., 2018). KRT7 could distinguish the precursor lesions of papillary renal cell tumors, mucinous tubular and spindle cell carcinomas (Szponar and Kovacs, 2012). The detailed information of CST6-related genes list is shown in Supplementary Table 4. These results indicated the conservative expression pattern 


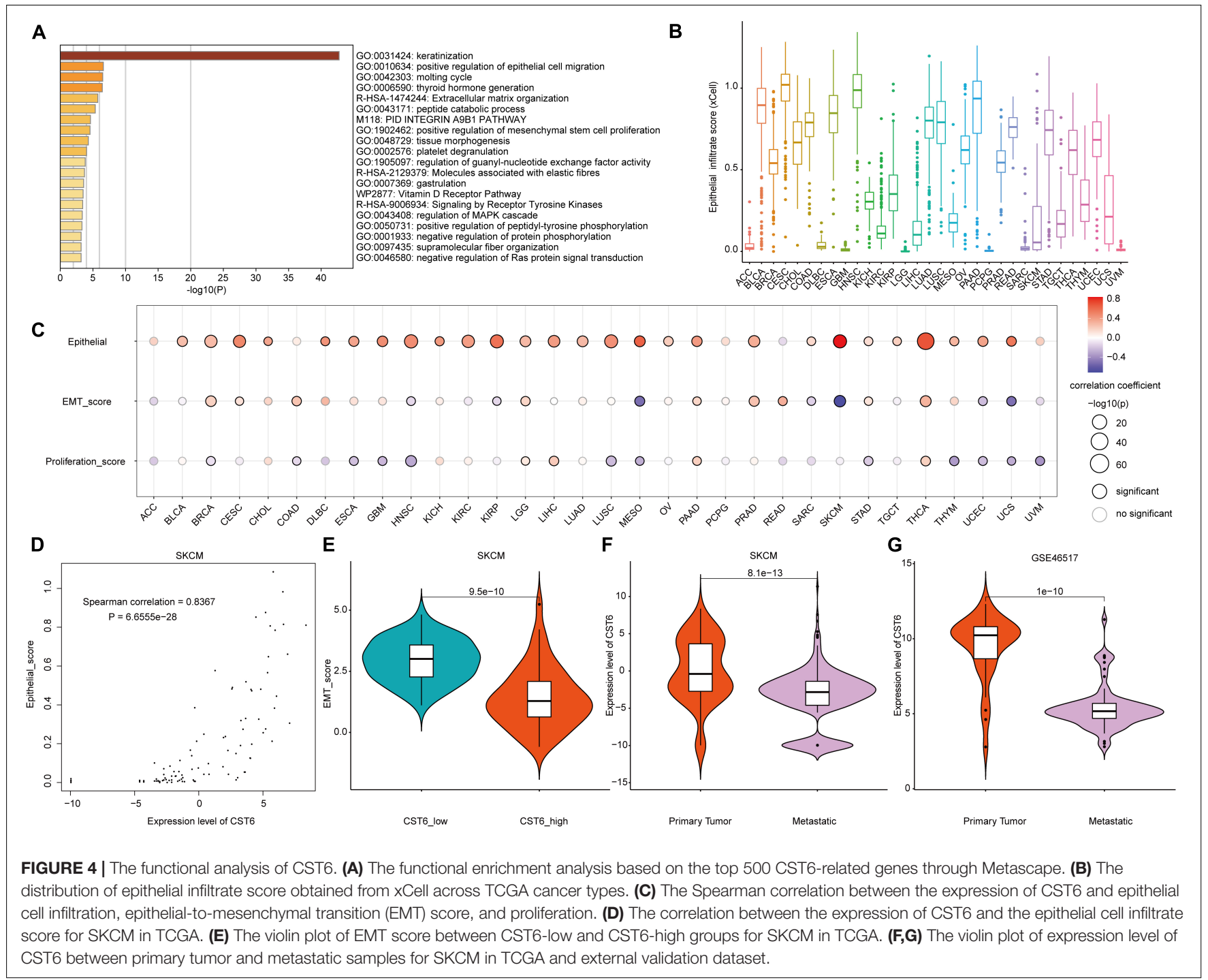

and important interactive function of CST6 in different types of cancer.

\section{The Cystatin E/M Expression Was Regulated by DNA Methylation}

CST6 expression has been previously associated with its epigenetic regulation by methylation of the promoter region in several cancer types (Rivenbark et al., 2006; Pulukuri et al., 2009; Peters et al., 2014). To explore the relationship between its expression and DNA methylation in TCGA datasets, we first quantified the methylation level of CST6 by averaging the CpG beta value in its promoter region. Then the Wilcoxon test was used to evaluate differentially methylated status between tumor and adjacent normal samples. In contrast to the expression pattern, we found that the methylation level of CST6 was significantly lower in BLCA, CESC, COAD, ESCA, HNSC, KIRP, LUAD, PAAD, READ, THCA, and UCEC tumor samples, while it was higher in BRCA, KIRC, liver hepatocellular carcinoma (LIHC), and prostate adenocarcinoma
(PRAD) (Wilcoxon test, $p$-value $<0.05$, Figure 3A). Next, we observed a significant negative correlation of CST6 methylation and its expression in more than half (21 out of 32, Pearson correlation $<0$ and $p$-value $<0.05$ ) of the cancer types (Figure 3B). The relationship of methylation and expression level of CST6 for the top correlated cancer types (BRCA, CHOL, KIRP, MESO, STAD, THCA, THYM, and USC, Pearson correlation $<-0.3$ and $p$-value $<0.01)$ is shown in Figure 3C. All these results further proved the capacity of DNA methylation in regulating the gene expression of CST6.

\section{Functional Analysis of Cystatin E/M in Cancer}

Taking advantage of integration of transcriptome and DNA methylation resource, we characterized the expression pattern and DNA methylation regulatory mechanism of CST6. To further investigate the function of CST6 across cancer types, we first obtained 500 CST6-related genes (Supplementary Table 4) for all TCGA tumor samples from the GEPIA2 "Correlation Analysis" 


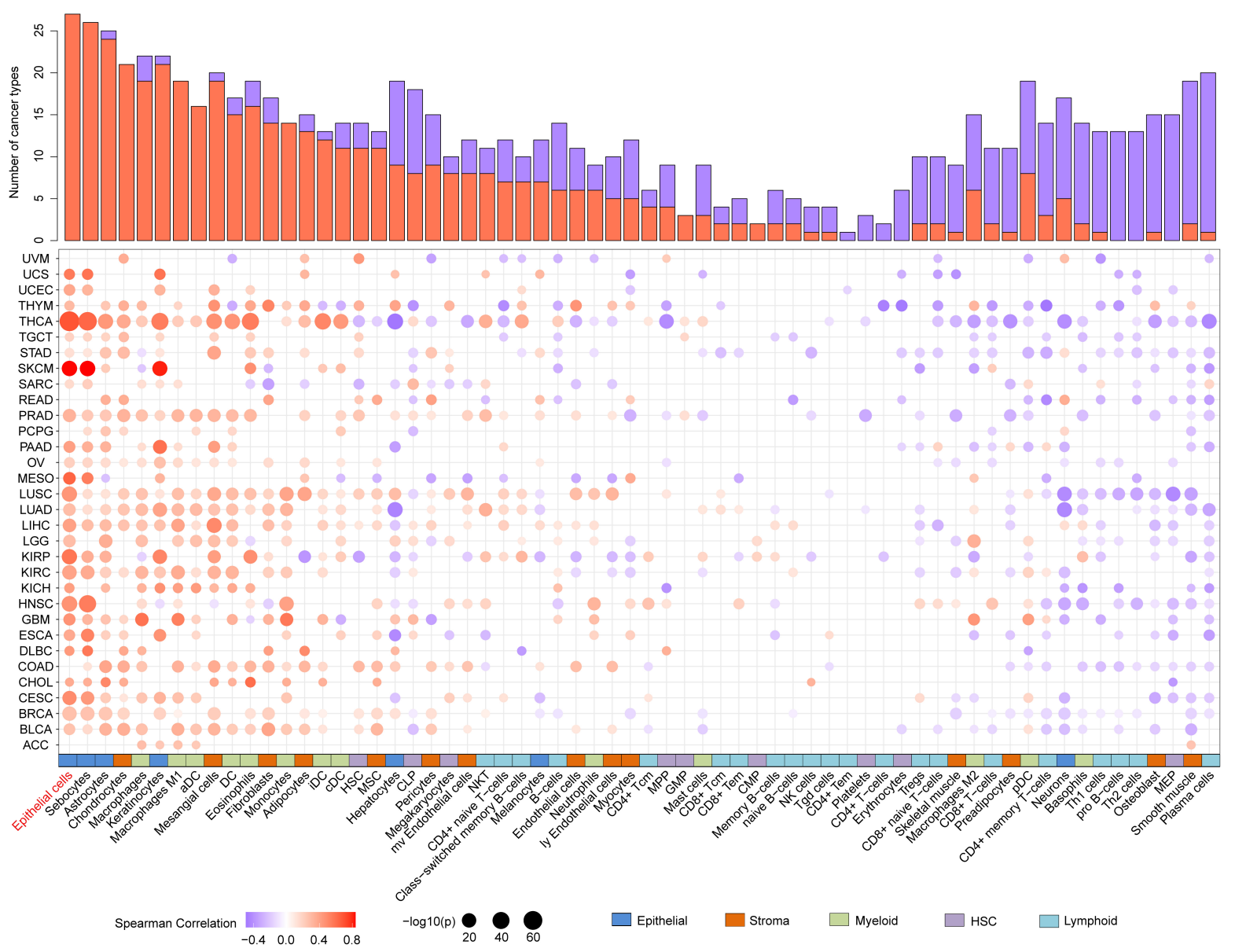

FIGURE 5 | Association analysis between the expression of CST6 and tumor microenvironment in TCGA datasets. Histogram height represents the number of cancer types with significant correlation between CST6 and cell infiltration score. Red- and purple-colored bubbles denote positive and negative correlation. Colored bar on the bottom denotes cell subgroup obtained from $x$ Cell.

module. Then, the functional enrichment analysis was performed through Metascape based on the CST6-related gene list. CST6related genes were significantly enriched in keratinization and positive regulation of epithelial cell migration (Figure 4A). Given that the strong correlation between keratin genes and epithelial cell has been reported (Heatley, 2002; Moll et al., 2008), we next explored the relationship of CST6 and epithelial cell in detail. The expression level of CST6 and epithelial cell infiltrate score were varied in cancer types (Figure 4B and Supplementary Figure 3), while the positive correlation between the two variables was observed in most cancers (Figure $4 \mathrm{C}$ and Supplementary Table 5).

Considering the fact that CST6 has been previously associated with the GO term "positive regulation of mesenchymal stem cell proliferation," we next explored the role that CST6 plays in EMT and proliferation. At first, we downloaded tumor purity for TCGA samples from a previous study (Thorsson et al., 2018). As the EMT score was significantly influenced by tumor purity (Supplementary Table 6), we estimated the relationship between EMT and CST6 using partial correlation to remove the confounder. In contrast to epithelial cell infiltration,
CST6 showed dual functional effects on EMT and proliferation (Figure 4C and Supplementary Table 5). Particularly, the correlation coefficients of epithelial cell and EMT score in SKCM were reversed (Figures 4D,E), which indicated a potential role of CST6 in the metastasis of melanoma. Given that SKCM has the maximum number of metastatic samples in TCGA, we next compared the expression level of CST6 between metastasis samples and primary tumor tissues. As shown in Figure 4F, the expression of CST6 was significantly higher in SKCM primary tumor tissues than that of metastatic samples. Consistent with this finding, a similar pattern was observed in another SKCM metastatic dataset (GSE46517, Figure 4G), which further proved the protective effect of CST6 in melanoma metastasis. Taken together, all these results suggest that CST6 was related to epithelial cell infiltration and tumor EMT process.

As evidence has shown the positive correlation between CST6 and epithelial cell, we next explored its relationship with other cells. As shown in Figure 5, the expression of CST6 was positively related to most epithelial cells, while the negative correlation between CST6 and plasma cell was observed in most cancer types. A previous study has found that CST6 promoter 
A

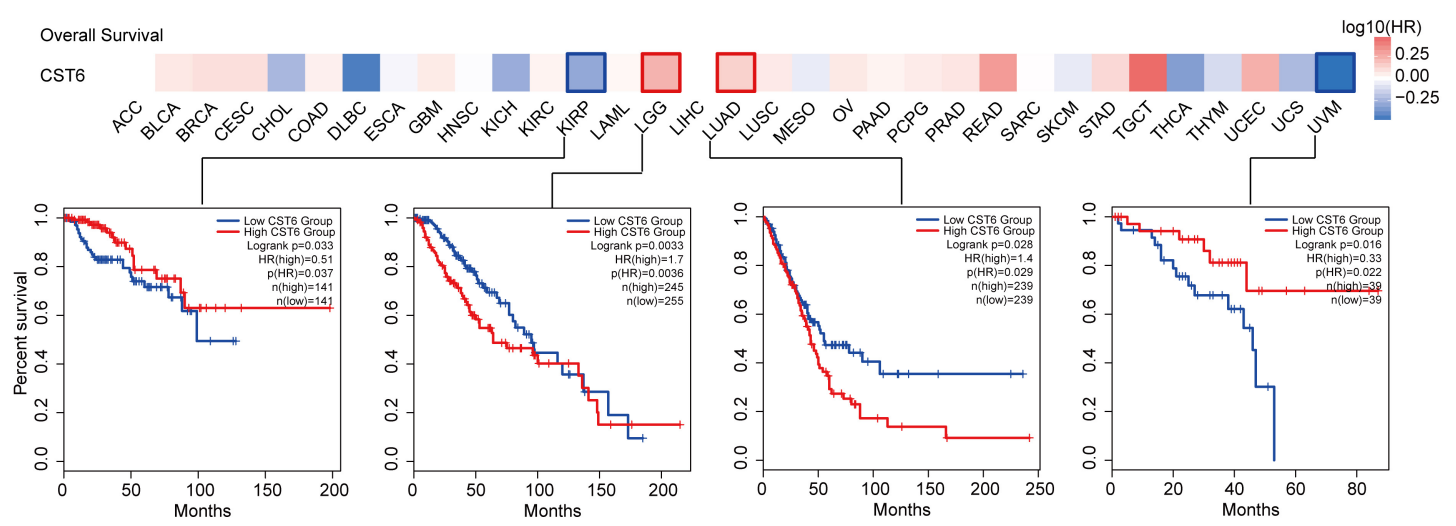

B

Disease Free Survival

CST6

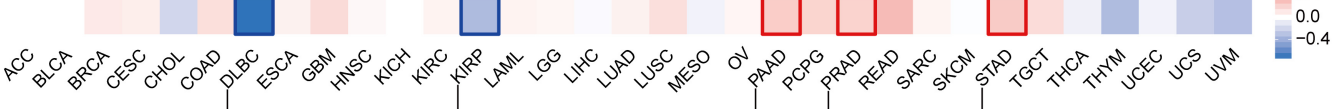
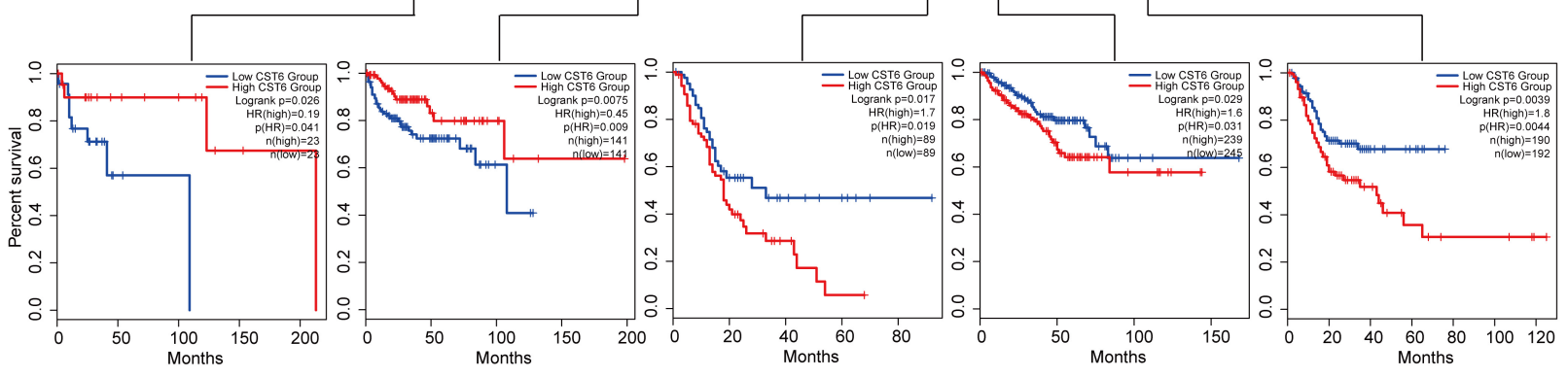

C
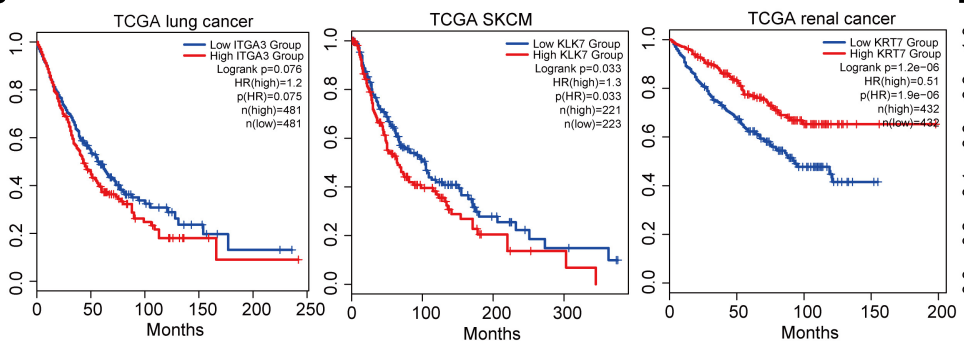

D

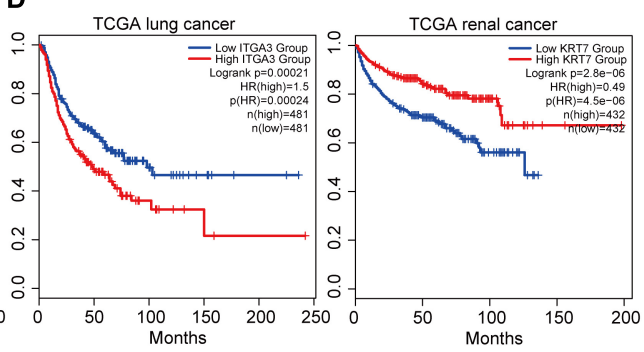

FIGURE 6 | Clinical association analysis between the expression of CST6 and survival prognosis across TCGA cancer types. (A) The survival map and Kaplan-Meier estimates of overall survival by CST6 expression in TCGA datasets. (B) The survival map and Kaplan-Meier estimates of disease-free survival by CST6 expression in TCGA datasets. (C) The Kaplan-Meier estimates of overall survival by ITGA3, KLK7, and KRT7 expression in TCGA lung cancer, melanoma, and renal cancer datasets. (D) The Kaplan-Meier estimates of disease-free survival by ITGA3 and keratin 7 (KRT7) expression in TCGA lung and renal cancer datasets.

is highly methylated in cfDNA of BRCA plasma cells but not in healthy samples (Chimonidou et al., 2013). These results suggest the potential regulatory roles of CST6 in the tumor microenvironment.

\section{Clinical Associations of Cystatin E/M in Cancer}

We next explored the critical efficiency of CST6 in the survival of tumor patients. Tumor samples were divided into highexpression and low-expression groups based on CST6 expression for each TCGA tumor type. Patients with a higher expression of CST6 had worse survival in brain lower-grade glioma (LGG), LUAD, PAAD, PRAD, and stomach adenocarcinoma (STAD)
(HR $>1$ and log-rank $p<0.05$, Figures 6A,B), while they indicated a favorable prognosis in KIRP, uveal melanoma (UVM), and diffuse large B-cell lymphoma (DLBC) $(\mathrm{HR}<1$ and $\log$-rank $p<0.05$, Figures $6 \mathbf{A}, \mathbf{B})$. The multivariate Cox regression model was also performed with several clinical factors (Supplementary Figure 4). In addition, the CST6related genes mentioned above (ITGA3, LKL7, and KRT7 corresponding to TCGA lung cancer, SKCM, and renal cancer separately) were found to be associated with clinical outcomes (Figures 6C,D). These results revealed the dual effects of CST6 on the survival of patient.

The association between epithelial and CST6 for SKCM has been examined herein before. Next, we explored whether these two important elements affected the clinical survival of 


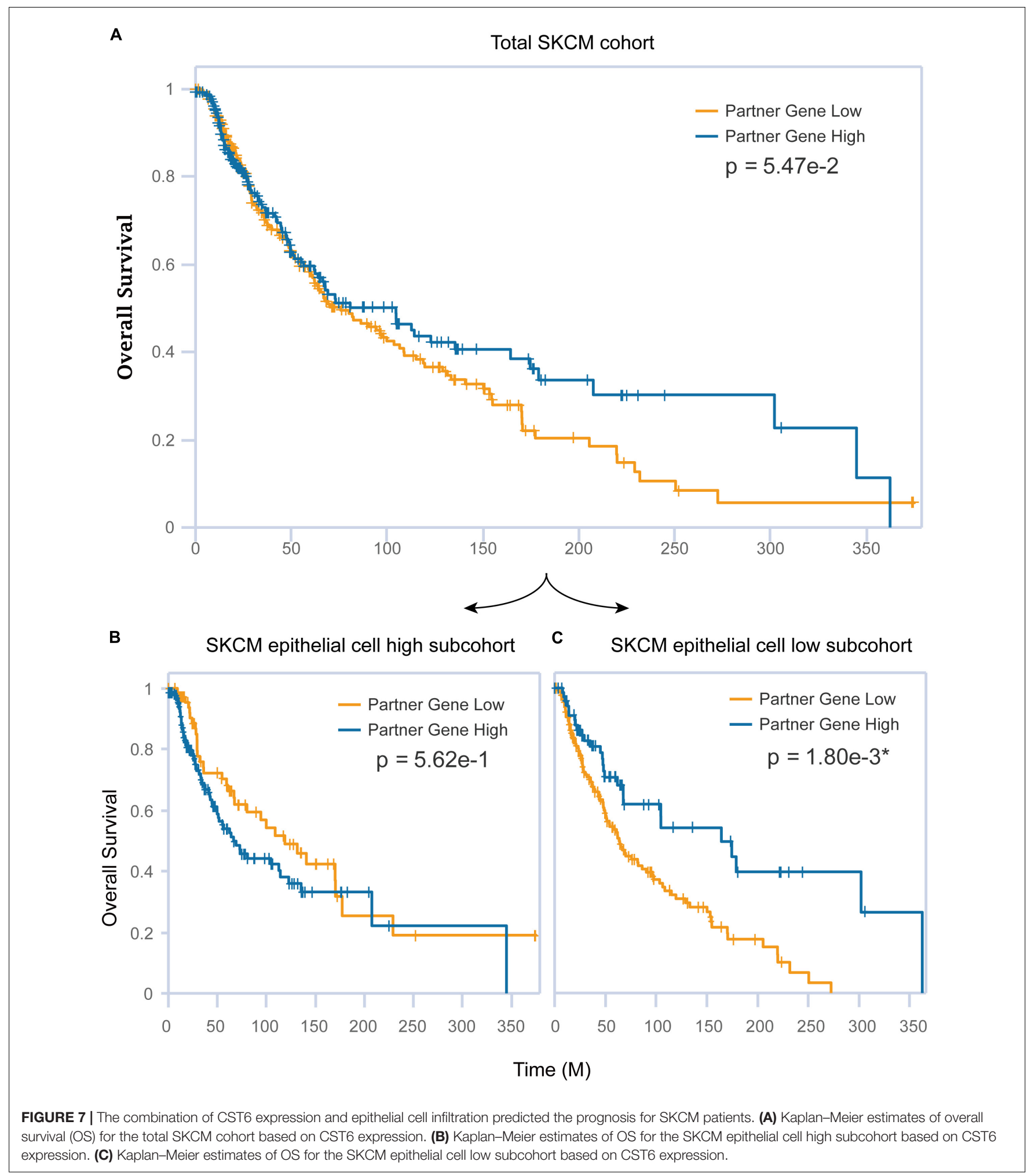

melanoma patients. The expression of CST6 could not well predict the prognosis of SKCM patients (log-rank $p=0.0547$, Figure 7A) using siGCD. Meanwhile, we found that the epithelial cell infiltration score of SKCM primary tumor was significantly higher than the metastatic patients (Wilcoxon test, $p$-value $<0.05$, Supplementary Figure 5A). Moreover, the expression of CST6 could serve as a protective factor for the clinical survival of metastatic patients, which indicates the 
important roles of epithelial cell and CST6 in SKCM survival (Supplementary Figures 5B,C). Thus, we took the epithelial marker from $x$ Cell into consideration. In the case of the epithelial cell low subcohort, the OS of patients with high CST6 expression showed significantly better than those with low scores, while the results did not occur in the epithelial cell high subcohort (Figures 7B,C). These results implied that the combination of molecular expression and cell infiltration could better predict the survival of cancer patients.

\section{DISCUSSION AND CONCLUSION}

The dual function of CST6 as both tumor suppressing and tumor promoting has been well appreciated (Lalmanach et al., 2021), but its global function and expression pattern in the development of cancer remain largely unknown. Here, we comprehensively characterized the expression pattern of CST6 in cancer from TCGA, and the result was verified from another large sample dataset. Consistent with prior knowledge, the expression of CST6 showed that it was downregulated with tumor-suppressing function, while it showed a reverse level with tumor-promoting function. Evidence has shown the EMT and metastasis functions of ITGA3 in lung cancer, which were similar to the function of CST6 (Li et al., 2020). Meanwhile, we observed a conservative correlation between CST6 and ITGA3 in two datasets (TCGA and MMDs), providing potential therapeutic targets for lung cancer. Apart from ITGA3, we also identified KLK7 and KRT7 as CST6-related genes in melanoma and renal cancer datasets. Overexpression of KLK7 induced a significant reduction in melanoma cell proliferation and colony formation (Delaunay et al., 2017). KRT7 has been proven to be an important biomarker for kidney cancer (Williamson et al., 2017). Taken together, these results indicate the conservative expression pattern and essential interactive function of CST6 in human tumors.

Given that the expression of CST6 exhibited epigenetic inactivation in special cancer types, we explored the relationship between DNA methylation and its expression across cancer types. We found that the expression of CST6 was globally regulated by DNA methylation, especially in BRCA, KIRP, MESO, STAD, THCA, and THYM cancer types. Although we revealed the essential roles of DNA methylation in regulating CST6 expression, we cannot explain its differential expression in some cancer types. Previously, two SNPs in the 5'UTR region of CST6 have been found to be associated with fluconazole susceptibility through a genome-wide association study (Guo et al., 2020). Thus, we counted the number of SNVs located in the CST6 gene region from cBioPortal. Thirteen cancer types with CST6 mutation were identified. Among them, SKCM has the highest alteration frequency (range from 0.19 to $1.36 \%$, Supplementary Figure 6A). To explore the effect of SNV on the alteration of CST6 expression, we search the PancanQTL to obtain the CST6related cis-eQTLs (within $1 \mathrm{Mb}$ from the gene transcriptional start site) (Gong et al., 2018). We found more than 30 cis-eQTLs in STAD and THCA, and the alternation of rs619701 could improve the expression level of CST6 in THCA (Supplementary Figures 6B,C and Supplementary Table 7). Integration of SNV data may provide a novel insight for understanding the regulatory mechanism of CST6 in cancer.

Moreover, we found that the expression of CST6 was globally positively correlated with epithelial cell infiltration, suggesting its important roles in the epithelium. Rivenbark et al. (2007) have observed a strong immunostaining phenomenon for CST6 in normal breast epithelial and myoepithelial cells, while it was negative in primary breast tumors. The relevance between CST6 and epithelium encourages us to further explore its relationship with EMT. As the EMT process plays an essential role in cancer metastasis (Brabletz et al., 2018), we found the protective function of CST6 in melanoma metastasis considering the negative correlation between CST6 and EMT score. Although the low-level internalization of CST6 that could affect the migration of melanoma cell has been proven (Wallin et al., 2017), we first revealed the potential mechanism between CST6 and EMT in the melanoma metastasis. Besides, we also found that the combination of epithelium infiltration and CST6 expression could well predict the survival of SKCM patients. Our results suggested the necessity to consider molecular and tumor microenvironment in tumor prognostics.

Our results have been partially limited by the nature of the datasets. Although the variation trend in expression and DNA methylation of CST6 was adverse in most cancer types, a few discordant events were also observed. For instance, the expression and DNA methylation level of CST6 were all upregulated in BRCA tumor samples. This may due to the unbalanced sample size between tumor and normal samples. The discordant of CST6 expression in transcriptome and proteome has also been observed (KIRC was the only cancer type that CST6 showed to be downregulated in both TCGA and CPTAC datasets). Meanwhile, there was a considerable number of lncRNA involved in the CST6-related genes. Thus, the posttranscriptional regulation like non-coding RNA may be another explanation, and this will be the further direction that we will analyze.

In summary, our comprehensive analysis of the expression pattern and dual functional effects of CST6 in pan-cancer level reveals its essential roles. The expression of CST6 was globally regulated by DNA methylation and related to epithelium infiltration. Particularly, CST6 performed a protective function in melanoma metastasis. Dysfunction of CST6 has also shown dual effects in clinical survival in different cancer types.

\section{DATA AVAILABILITY STATEMENT}

The datasets presented in this study can be found in online repositories. The names of the repository/repositories and accession number(s) can be found in the article/Supplementary Material.

\section{AUTHOR CONTRIBUTIONS}

$\mathrm{KL}, \mathrm{ZM}$, and $\mathrm{XB}$ conceived, designed the experiments, finalized, and submitted the manuscript. DX, SD, MC, XY, HW, DQ, and 
ZX analyzed the data. DX, SD, and MC drafted the manuscript. All authors have read and approved the final manuscript.

\section{FUNDING}

This work was supported by the Hainan Provincial Natural Science Foundation of China (820RC637 and 820RC627), National Natural Science Foundation of China

\section{REFERENCES}

Ai, L., Kim, W. J., Kim, T. Y., Fields, C. R., Massoll, N. A., Robertson, K. D., et al. (2006). Epigenetic silencing of the tumor suppressor cystatin M occurs during breast cancer progression. Cancer Res. 66, 7899-7909. doi: 10.1158/0008-5472. CAN-06-0576

Aran, D., Hu, Z., and Butte, A. J. (2017). xCell: digitally portraying the tissue cellular heterogeneity landscape. Genome Biol. 18, 1-14. doi: 10.1186/s13059017-1349-1

Brabletz, T., Kalluri, R., Nieto, M. A., and Weinberg, R. A. (2018). EMT in cancer. Nat. Rev. Cancer 18, 128-134. doi: 10.1038/nrc.2017.118

Briggs, J. J., Haugen, M. H., Johansen, H. T., Riker, A. I, Abrahamson, M., Fodstad, $\varnothing$, et al. (2010). Cystatin E/M suppresses legumain activity and invasion of human melanoma. BMC Cancer 10:17. doi: 10.1186/1471-2407-10-17

Chandrashekar, D. S., Bashel, B., Balasubramanya, S. A. H., Creighton, C. J., PonceRodriguez, I., Chakravarthi, B. V. S. K., et al. (2017). UALCAN: a portal for facilitating tumor subgroup gene expression and survival analyses. Neoplasia (United States) 19, 649-658. doi: 10.1016/j.neo.2017.05.002

Chimonidou, M., Tzitzira, A., Strati, A., Sotiropoulou, G., Sfikas, C., Malamos, N., et al. (2013). CST6 promoter methylation in circulating cell-free DNA of breast cancer patients. Clin. Biochem. 46, 235-240. doi: 10.1016/j.clinbiochem.2012. 09.015

Cui, X., Han, L., Liu, Y., Li, Y., Sun, W., Song, B., et al. (2021). siGCD: a web server to explore survival interaction of genes, cells and drugs in human cancers. Brief. Bioinform. bbab058. doi: 10.1093/bib/bbab058 [Epub ahead of print].

Delaunay, T., Deschamps, L., Haddada, M., Walker, F., Soosaipillai, A., Soualmia, F., et al. (2017). Aberrant expression of kallikrein-related peptidase 7 is correlated with human melanoma aggressiveness by stimulating cell migration and invasion. Mol. Oncol. 11, 1330-1347. doi: 10.1002/1878-0261.1 2103

Gong, J., Mei, S., Liu, C., Xiang, Y., Ye, Y., Zhang, Z., et al. (2018). PancanQTL: systematic identification of cis -eQTLs and trans -eQTLs in 33 cancer types. Nucleic Acids Res. 46, D971-D976. doi: 10.1093/nar/gkx861

Guo, X., Zhang, R., Li, Y., Wang, Z., Ishchuk, O. P., Ahmad, K. M., et al. (2020). Understand the genomic diversity and evolution of fungal pathogen Candida glabrata by genome-wide analysis of genetic variations. Methods 176, 82-90. doi: 10.1016/j.ymeth.2019.05.002

Haddada, M., Draoui, H., Deschamps, L., Walker, F., Delaunay, T., Brattsand, M., et al. (2018). Kallikrein-related peptidase 7 overexpression in melanoma cells modulates cell adhesion leading to a malignant phenotype. Biol. Chem. 399, 1099-1105. doi: 10.1515/hsz-2017-0339

Heatley, M. K. (2002). Keratin expression in human tissues and neoplasms [1]. Histopathology 41, 365-366. doi: 10.1046/j.1365-2559.2002.15261.x

Hinshaw, D. C., and Shevde, L. A. (2019). The tumor microenvironment innately modulates cancer progression. Cancer Res. 79, 4557-4566. doi: 10.1158/00085472.CAN-18-3962

Hosokawa, M., Kashiwaya, K., Eguchi, H., Ohigashi, H., Ishikawa, O., Furihata, M., et al. (2008). Over-expression of cysteine proteinase inhibitor cystatin 6 promotes pancreatic cancer growth. Cancer Sci. 99, 1626-1632. doi: 10.1111/ j.1349-7006.2008.00869.X

Jin, L., Zhang, Y., Li, H., Yao, L., Fu, D., Yao, X., et al. (2012). Differential secretome analysis reveals CST6 as a suppressor of breast cancer bone metastasis. Cell Res. 22, 1356-1373. doi: 10.1038/cr.2012.90
(32160152), Major Science and Technology Program of Hainan Province (ZDKJ202003), and Hainan Province Clinical Medical Center.

\section{SUPPLEMENTARY MATERIAL}

The Supplementary Material for this article can be found online at: https://www.frontiersin.org/articles/10.3389/fgene. 2021.733211/full\#supplementary-material

Keppler, D. (2006). Towards novel anti-cancer strategies based on cystatin function. Cancer Lett. 235, 159-176. doi: 10.1016/j.canlet.2005.04.001

Lalmanach, G., Kasabova-Arjomand, M., Lecaille, F., and Saidi, A. (2021). Cystatin $\mathrm{m} / \mathrm{e}$ (Cystatin 6): a janus-faced cysteine protease inhibitor with both tumorsuppressing and tumor-promoting functions. Cancers (Basel). 13, 1-18. doi: $10.3390 /$ cancers 13081877

Leek, J. T., Johnson, W. E., Parker, H. S., Jaffe, A. E., and Storey, J. D. (2012). The sva package for removing batch effects and other unwanted variation in high-throughput experiments. Bioinformatics 28, 882-883. doi: 10.1093/ bioinformatics/bts034

Li, Q., Ma, W., Chen, S., Tian, E. C., Wei, S., Fan, R. R., et al. (2020). High integrin a3 expression is associated with poor prognosis in patients with non-small cell lung cancer. Transl. Lung Cancer Res. 9, 1361-1378. doi: 10.21037/tlcr-19-633

Li, Q., Zheng, Z. C., Ni, C. J., Jin, W. X., Jin, Y. X., Chen, Y., et al. (2018). Correlation of cystatin E/M with clinicopathological features and prognosis in triple-negative breast cancer. Ann. Clin. Lab. Sci. 48, 40-44.

Li, T., Fu, J., Zeng, Z., Cohen, D., Li, J., Chen, Q., et al. (2021). TIMER2.0 for analysis of tumor-infiltrating immune cells. Nucleic Acids Res. 48, W509-W514. doi: 10.1093/NAR/GKAA407

Lim, S. B., Chua, M. L. K., Yeong, J. P. S., Tan, S. J., Lim, W.-T., and Lim, C. T. (2019). Pan-cancer analysis connects tumor matrisome to immune response. NPJ Precis. Oncol. 3:15. doi: 10.1038/s41698-019-0087-0

Mak, M. P., Tong, P., Diao, L., Cardnell, R. J., Gibbons, D. L., William, W. N., et al. (2016). A patient-derived, pan-cancer EMT signature identifies global molecular alterations and immune target enrichment following epithelial-tomesenchymal transition. Clin. Cancer Res. 22, 609-620. doi: 10.1158/10780432.CCR-15-0876

Moll, R., Divo, M., and Langbein, L. (2008). The human keratins: biology and pathology. Histochem. Cell Biol. 129, 705-733. doi: 10.1007/s00418-008-0435-6

Morris, M. R., Ricketts, C., Gentle, D., Abdulrahman, M., Clarke, N., Brown, M., et al. (2010). Identification of candidate tumour suppressor genes frequently methylated in renal cell carcinoma. Oncogene 29, 2104-2117. doi: 10.1038/onc 2009.493

Oler, G., Camacho, C. P., Hojaij, F. C., Michaluart, P., Riggins, G. J., and Cerutti, J. M. (2008). Gene expression profiling of papillary thyroid carcinoma identifies transcripts correlated with BRAF mutational status and lymph node metastasis. Clin. Cancer Res. 14, 4735-4742. doi: 10.1158/1078-0432.CCR-07-4372

Peters, I., Dubrowinskaja, N., Abbas, M., Seidel, C., Kogosov, M., Scherer, R. et al. (2014). DNA methylation biomarkers predict progression-free and overall survival of metastatic renal cell cancer (mRCC) treated with antiangiogenic therapies. PLoS One 9:e91440. doi: 10.1371/journal.pone.0091440

Pulukuri, S. M., Gorantla, B., Knost, J. A., and Rao, J. S. (2009). Frequent loss of cystatin $\mathrm{E} / \mathrm{M}$ expression implicated in the progression of prostate cancer. Oncogene 28, 2829-2838. doi: 10.1038/onc.2009.134

Qiu, J., Ai, L., Ramachandran, C., Yao, B., Gopalakrishnan, S., Fields, C. R., et al. (2008). Invasion suppressor cystatin E/M (CST6): high-level cell type-specific expression in normal brain and epigenetic silencing in gliomas. Lab. Invest. 88, 910-925. doi: 10.1038/labinvest.2008.66

Rivenbark, A. G., Jones, W. D., and Coleman, W. B. (2006). DNA methylationdependent silencing of CST6 in human breast cancer cell lines. Lab. Invest. 86, 1233-1242. doi: 10.1038/labinvest.3700485

Rivenbark, A. G., Livasy, C. A., Boyd, C. E., Keppler, D., and Coleman, W. B. (2007). Methylation-dependent silencing of CST6 in primary human breast tumors and 
metastatic lesions. Exp. Mol. Pathol. 83, 188-197. doi: 10.1016/j.yexmp.2007.03. 008

Sato, E., Yano, N., Fujita, Y., and Imafuku, S. (2020). Interleukin-17A suppresses granular layer formation in a 3-D human epidermis model through regulation of terminal differentiation genes. J. Dermatol. 47, 390-396. doi: 10.1111/13468138.15250

Schagdarsurengin, U., Pfeifer, G. P., and Dammann, R. (2007). Frequent epigenetic inactivation of cystatin M in breast carcinoma. Oncogene 26, 3089-3094. doi: 10.1038/sj.onc. 1210107

Shridhar, R., Zhang, J., Song, J., Booth, B. A., Kevil, C. G., Sotiropoulou, G., et al. (2004). Cystatin M suppresses the malignant phenotype of human MDA-MB435 S cells. Oncogene 23, 2206-2215. doi: 10.1038/sj.onc. 1207340

Szponar, A., and Kovacs, G. (2012). Expression of KRT7 and WT1 differentiates precursor lesions of Wilms' tumours from those of papillary renal cell tumours and mucinous tubular and spindle cell carcinomas. Virchows Arch. 460, 423427. doi: 10.1007/s00428-012-1209-z

Tang, Z., Kang, B., Li, C., Chen, T., and Zhang, Z. (2019). GEPIA2: an enhanced web server for large-scale expression profiling and interactive analysis. Nucleic Acids Res. 47, W556-W560. doi: 10.1093/nar/gkz430

Thorsson, V., Gibbs, D. L., Brown, S. D., Wolf, D., Bortone, D. S., Ou Yang, T.H., et al. (2018). The immune landscape of cancer. Immunity 48, 812-830.e14. doi: 10.1016/j.immuni.2018.03.023

Turk, V., and Bode, W. (1991). The cystatins: protein inhibitors of cysteine proteinases. FEBS Lett. 285, 213-219. doi: 10.1016/0014-5793(91)8 0804-C

Veena, M. S., Lee, G., Keppler, D., Mendonca, M. S., Redpath, J. L., Stanbridge, E. J., et al. (2008). Inactivation of the cystatin E/M tumor suppressor gene in cervical cancer. Genes Chromosomes Cancer 47, 740-754. doi: 10.1002/gcc.20576

Vigneswaran, N., Wu, J., and Zacharias, W. (2003). Upregulation of cystatin $\mathrm{M}$ during the progression of oropharyngeal squamous cell carcinoma from primary tumor to metastasis. Oral Oncol. 39, 559-568. doi: 10.1016/S13688375(03)00038-1

Wallin, H., Apelqvist, J., Andersson, F., Ekström, U., and Abrahamson, M. (2017). Low-level internalization of cystatin E/M affects legumain activity and migration of melanoma cells. J. Biol. Chem. 292, 14413-14424. doi: 10.1074/jbc. M117.776138

Williamson, S. R., Gadde, R., Trpkov, K., Hirsch, M. S., Srigley, J. R., Reuter, V. E., et al. (2017). Diagnostic criteria for oncocytic renal neoplasms: a survey of urologic pathologists. Hum. Pathol. 63, 149-156. doi: 10.1016/j.humpath.2017. 03.004
Wu, T., and Dai, Y. (2017). Tumor microenvironment and therapeutic response. Cancer Lett. 387, 61-68. doi: 10.1016/j.canlet.2016.01.043

Zeeuwen, P. L. J. M., van Vlijmen-Willems, I. M. J. J., Cheng, T., Rodijk-Olthuis, D., Hitomi, K., Hara-Nishimura, I., et al. (2010). The cystatin M/E-cathepsin $\mathrm{L}$ balance is essential for tissue homeostasis in epidermis, hair follicles, and cornea. FASEB J. 24, 3744-3755. doi: 10.1096/fj.10-155879

Zhang, J., Shridhar, R., Dai, Q., Song, J., Barlow, S. C., Yin, L., et al. (2004). Cystatin m: a novel candidate tumor suppressor gene for breast cancer. Cancer Res. 64, 6957-6964. doi: 10.1158/0008-5472.CAN-04-0819

Zhang, Z., Jing, J., Ye, Y., Chen, Z., Jing, Y., Li, S., et al. (2020). Characterization of the dual functional effects of heat shock proteins (HSPs) in cancer hallmarks to aid development of HSP inhibitors. Genome Med. 12, 1-16. doi: 10.1186/ s13073-020-00795-6

Zhong, S., Fields, C. R., Su, N., Pan, Y. X., and Robertson, K. D. (2007). Pharmacologic inhibition of epigenetic modifications, coupled with gene expression profiling, reveals novel targets of aberrant DNA methylation and histone deacetylation in lung cancer. Oncogene 26, 2621-2634. doi: 10.1038/sj. onc. 1210041

Zhou, Y., Zhou, B., Pache, L., Chang, M., Khodabakhshi, A. H., Tanaseichuk, O., et al. (2019). Metascape provides a biologist-oriented resource for the analysis of systems-level datasets. Nat. Commun. 10:1523. doi: 10.1038/s41467-019-09 234-6

Conflict of Interest: The authors declare that the research was conducted in the absence of any commercial or financial relationships that could be construed as a potential conflict of interest.

Publisher's Note: All claims expressed in this article are solely those of the authors and do not necessarily represent those of their affiliated organizations, or those of the publisher, the editors and the reviewers. Any product that may be evaluated in this article, or claim that may be made by its manufacturer, is not guaranteed or endorsed by the publisher.

Copyright (c) $2021 \mathrm{Xu}$, Ding, Cao, Yu, Wang, Qiu, Xu, Bi, Mu and Li. This is an open-access article distributed under the terms of the Creative Commons Attribution License (CC BY). The use, distribution or reproduction in other forums is permitted, provided the original author(s) and the copyright owner(s) are credited and that the original publication in this journal is cited, in accordance with accepted academic practice. No use, distribution or reproduction is permitted which does not comply with these terms. 